\title{
Quantitative Assessment on the Severity Degree of Alzheimer Dementia by Algebraic Bigdata Analysis on Cortical Thickness Profiles of Human Brains
}

\section{Sangyeol Kim}

Daegu Gyeongbuk Institute of Science and Technology

\section{Seongjun Park}

Daegu Gyeongbuk Institute of Science and Technology

Iksoo Chang ( $\nabla$ iksoochang@dgist.ac.kr)

Daegu Gyeongbuk Institute of Science and Technology

\section{Research}

Keywords: Alzheimer's disease, dementia, mild cognitive impairment, severity degree, cortical thickness

Posted Date: December 11th, 2020

DOI: https://doi.org/10.21203/rs.3.rs-125102/v1

License: (c) (i) This work is licensed under a Creative Commons Attribution 4.0 International License.

Read Full License 
1 Quantitative assessment on the severity degree of Alzheimer dementia

2 by algebraic bigdata analysis on cortical thickness profiles of human

3

4

5

6

7

$8{ }^{1}$ Department of Brain and Cognitive Sciences, Daegu Gyeongbuk Institute of Science

9 and Technology, Daegu, Korea

* Data used in preparation of this article were obtained from the Alzheimer's Disease

20 Neuroimaging Initiative (ADNI) database (adni.loni.usc.edu). As such, the

21 investigators within the ADNI contributed to the design and implementation of ADNI

22 and/or provided data but did not participate in analysis or writing of this report. A

23 complete listing of $\mathrm{ADNI}$ investigators can be found at: http://adni.loni.usc.edu/wp-

24 content/uploads/how_to_apply/ADNI_Acknowledgement_List.pdf. 


\section{Abstract}

2 Background: Alzheimer disease(AD) affects profoundly the quality of human life.

3 Quantifying the severity degree of $A D$ for an individual person is critical for the early

4 diagnose and prescription for delaying the further dementia progression. However, the

5 quantitative diagnose for human subjects of the mild cognitively impairment $(\mathrm{MCl})$ or

6 AD with the different degree of dementia severity is still a difficult task due to both the

7 very broad distribution of dementia severities and the lack of good quantitative

8 determinant to assess it.

9 Methods: We performed the bigdata analysis of cortical thickness of 1516 human

10 brain images. Instead of dealing with cortical thickness at all 327,684 vertices on the

11 whole cortex of a human brain from MRI, we extracted the essential cortical thickness

12 data at a few hundred vertices to distinguish efficiently $A D, M C l$, and cognitively normal

13 (CN) cohorts each other. We learned a statistical score matrix and a covariance

14 correlation matrix of cortical thickness profile between human subjects as a set of

15 classifier and predictor for diagnosing the dementia states of $A D$.

16 Results: The subjects with AD were recognized with more than $91 \%$ accuracy in self-

17 recognition test, and the independent validation subjects with $A D$ were predicted 18 correctly with more than $82 \%$ accuracy in stratified 3 -fold cross validation test. Given 19 a new person for diagnosing and provided with the covariance correlation matrix 20 developed in this study as a predictor, more importantly one could estimate the 21 personalized severity degree of dementia in terms of a quantitative value, ranging from

220 for the basin of $\mathrm{CN}$ state to 1 for the basin of $\mathrm{AD}$ state. Also, one could sort out the 23 broad spectrum of the severity of dementia for $\mathrm{MCl}$ subjects in that whether they are 24 prone to $\mathrm{CN}$ or how much they are progressed toward $\mathrm{AD}$. 
1 Conclusions: Here, a new approach of algebraic bigdata analysis is presented for the

2 quantitative assessment on both the severity degree of $A D$ and the systematic

3 classification of three cohorts based on cortical thickness data of 1516 human brain

4 images from MRI. This new approach can facilitate the better diagnose of AD with the

5 different degree of dementia.

6

7 Keywords: Alzheimer's disease, dementia, mild cognitive impairment, severity 8 degree, cortical thickness.

9 


\section{Introduction}

2 Alzheimer's disease $(A D)$ is one of the most well-known neurodegenerative diseases,

3 and it profoundly affects the quality of human life. Various causes of AD are known, 4 and clinical treatments for preventing or delaying the progression of AD are currently 5 practiced. The precise diagnosis of $A D$ requires not only the systematic identification 6 of cohorts that classify the different stages of brain progression toward AD but also the 7 estimation of the severity degree of $A D$ for a given individual [1-3]. The symptoms of

8 AD appear in various forms in the human body, behavior, and cognition, yet the direct 9 anatomical evidences appear in the structural change within the brain cortex [4-7]. 10 Among these evidences is the degradation of the cortical thickness of the human brain, 11 which is one of the imprints of AD. Such physical change can be monitored through 12 the neuro-image analysis, for example the magnetic resonance image (MRI) analysis 13 of the brain [8-13].

14 The anatomical degradation of the cortical thickness becomes more pronounced as 15 the degree of dementia becomes greater $[9,13]$. Therefore, there is a rationale for taking advantage of the degree of cortical degradation for determining the dementia cohort and estimating the severity of dementia progression. Given the big data information of cortical thickness for a single subject, however, we are faced with several obstacles to overcome in accomplishing abovementioned tasks. First, we noted that the person-to-person fluctuations in cortical thickness of an individual may overwhelm the degradation in cortical thickness. In clinical cases, we frequently observed that the average cortical thickness of some cognitively normal people is

23 thinner than that of people with $A D$, which appears to contrast conventional view. The 24 second obstacle is the ambiguity in what we should do if two different cohorts have a 25 difference in the cortical thickness in brain regions that have little to do with AD. In 
1 principle, we should construct some determinants for distinguishing a cognitively

2 normal person from a person with AD based on cortical thickness, but in practice, we

3 are confronted with the differences in cortical thickness in regions of the cortex that

4 are unrelated to the pathogenesis of $A D$. We noted that the abundant existence of

5 such unrelated regions is an intrinsic source that increases the uncertainty of the

6 dementia determinants and hinders the appropriate construction of good classifier and

7 predictor for $A D$.

8 In this study, instead of dealing with all 327,684 vertices point on the whole cortex of

9 a human brain, we strived to overcome the before mentioned obstacles and 10 demonstrated that the consideration of a few hundred essential vertices were enough

11 for distinguishing $\mathrm{CN}, \mathrm{MCl}, \mathrm{AD}$ cohorts each other. With cortical thickness data at

12 these essential vertices of 1006 human brain images for control and 510 human brain

13 images for independent validation, we defined the statistical score matrix and the

14 covariance correlation matrix between human subjects as a new set of classifier and 15 predictor for AD. Unlike the conventional view that the degradation of the cortical 16 thickness of human brain was responsible for $A D$, the singular valued decomposition 17 analysis of the statistical score matrix developed in this study revealed that the 18 simultaneous consideration of both thinner and thicker cortical regions together 19 compared to those of $\mathrm{CN}$ are important and necessary for the precise diagnose of the 20 severity of $A D$.

\section{Methods}

23 Preparation of cortical thickness data from MRI of 1522 human brain 24 images from ADNI 
1 We selected 274 individuals (human subjects) who were identified as CN, 265

2 individuals with $\mathrm{MCl}, 125$ individuals with $\mathrm{AD}$ from the ADNI-2 study of ADNI, and 97

3 with $\mathrm{MCl}$ from the ADNI-GO study of ADNI. A human brain image-data set of $1522 \mathrm{MR}$

4 images from a total of 761 subjects was constructed, for each of which both 1.2-mm

5 sagittal Magnetization Prepared Rapid Gradient Echo (MPRAGE) and

6 MPRAGE_SENSE2 images were taken separately.

8 Partition 1516 MR images of human brains into four groups and determine

9 the essential region-of-interest vertices for each group

10 We performed the FreeSurfer analysis to obtain the cortical thickness data at 327,684

11 vertices on the cortex of a human brain $[14,15]$. The cortical thickness at each vertex

12 ranges from $0 \mathrm{~mm}$ to $5 \mathrm{~mm}$. After eliminating those vertices at which cortical thickness

13 information was missing for any one of the 1522 MR images of human brains in the

14 ADNI data set, 276,825 common vertices whose cortical thickness values are

15 available for all 1522 MR images were selected for our study. The average cortical

16 thickness over 276,825 vertices for each brain images was evaluated, and we divided

171516 values of average thickness into four groups (A-D) of different windows of 18 average thickness except 6 values of that run out-of-bounds. Demographic 19 characteristics of the average cortical thickness of the four groups are listed in Table 201.

Table 1. Demographic Characteristics of the groups

\begin{tabular}{cccc}
\hline Group A & $\mathrm{CN}$ & $\mathrm{MCl}$ & $\mathrm{AD}$ \\
\hline $\mathrm{N}^{*}$ & 136 & 155 & 13 \\
female (\%) & 60.3 & 53.5 & 30.8
\end{tabular}




\begin{tabular}{|c|c|c|c|}
\hline Age, Mean (SD) & $72.5(5.3)$ & $72.1(5.1)$ & $78.1(5.0)$ \\
\hline$<>^{\dagger}$, Mean (SD) & $2.4580(0.0477)$ & $2.4549(0.0415)$ & $2.4676(0.0539)$ \\
\hline Group B & $\mathrm{CN}$ & $\mathrm{MCl}$ & $A D$ \\
\hline $\mathrm{N}$ & 212 & 262 & 67 \\
\hline female (\%) & 62.7 & 43.5 & 41.8 \\
\hline Age, Mean (SD) & $73.0(5.2)$ & 73.7 (5.3) & 76.7 (6.3) \\
\hline$<\mathrm{t}>$, Mean (SD) & $2.3516(0.0293)$ & $2.3429(0.0266)$ & $2.3491(0.0269)$ \\
\hline Group C & $\mathrm{CN}$ & $\mathrm{MCl}$ & $A D$ \\
\hline $\mathrm{N}$ & 159 & 209 & 84 \\
\hline female (\%) & 46.5 & 34.0 & 52.4 \\
\hline Age, Mean (SD) & $74.0(5.7)$ & $75.3(5.8)$ & $76.9(5.2)$ \\
\hline$<\mathrm{t}>$, Mean (SD) & $2.2566(0.0290)$ & $2.2573(0.0278)$ & $2.2455(0.0298)$ \\
\hline Group D & $\mathrm{CN}$ & $\mathrm{MCl}$ & $\overline{A D}$ \\
\hline $\mathrm{N}$ & 40 & 96 & 83 \\
\hline female (\%) & 22.5 & 35.4 & 26.5 \\
\hline Age, Mean (SD) & $78.1(6.5)$ & $76.2(5.7)$ & $77.4(6.6)$ \\
\hline$<\mathrm{t}>$, Mean $(\mathrm{SD})$ & $2.1514(0.0389)$ & $2.1448(0.0433)$ & $2.1373(0.0520)$ \\
\hline
\end{tabular}

${ }^{*}$ Number of MRI images; ${ }^{\dagger}$ Average cortical thickness over the 276,825 vertices. AD, Alzheimer's disease; $\mathrm{CN}$, cognitively normal; $\mathrm{MCl}$, mild cognitive impairment.

1 In order to assign subjects from each $\mathrm{CN}, \mathrm{MCl}$, and $\mathrm{AD}$ cohort into one of the four 2 groups (A-D) of average cortical thickness, we employed the $Z$ score criteria in 3 selecting the region-of-interest (ROI) vertices and the essential ROI vertices on the 4 cortex at which the distribution of cortical thickness of the $\mathrm{CN}$ cohort is distinguished 5 from that of the AD cohort within each group of average cortical thickness. A similar 6 procedure is repeated for distinguishing the $\mathrm{CN}$ cohort from the $\mathrm{MCl}$ cohort and also 7 the $\mathrm{MCl}$ cohort from the $\mathrm{AD}$ cohort: 
$1 \quad Z_{p}^{\mathrm{CN}-\mathrm{MCl}}=\frac{\left\langle t_{p, h \in \mathrm{CN}}\right\rangle-\left\langle t_{p, h \in \mathrm{MCl}}\right\rangle}{\sqrt{\frac{\sigma_{p, h \in \mathrm{CN}}^{2}}{n_{p, h \in \mathrm{CN}}}+\frac{\sigma_{p, h \in \mathrm{MCl}}^{2}}{n_{p, h \in \mathrm{MCl}}}}}, Z_{p}^{\mathrm{CN}-\mathrm{AD}}=\frac{\left\langle t_{p, h \in \mathrm{CN}}\right\rangle-\left\langle t_{p, h \in \mathrm{AD}}\right\rangle}{\sqrt{\frac{\sigma_{p, h \in \mathrm{CN}}^{2}}{n_{p, h \in \mathrm{CN}}}+\frac{\sigma_{p, h \in \mathrm{AD}}^{2}}{n_{p, h \in \mathrm{AD}}}}}, Z_{p}^{\mathrm{MCl}-\mathrm{AD}}=$

$2 \frac{\left\langle t_{p, h \in \mathrm{MCI}}\right\rangle-\left\langle t_{p, h \in \mathrm{AD}}\right\rangle}{\sqrt{\frac{\sigma_{p, h \in \mathrm{MCI}}^{2}}{n_{p, h \in \mathrm{MCl}}}+\frac{\sigma_{p, h \in \mathrm{AD}}^{2}}{n_{p, h \in \mathrm{AD}}}}}$

3 Here, $\left\langle t_{p, h \in k}\right\rangle$ is the average cortical thickness at a vertex point $p$ averaged over the

4 subject $h$ who belongs to the $k$ (one of $\mathrm{CN}, \mathrm{MCl}, \mathrm{AD}$ ) cohort, and $\sigma_{p, h \in k}$ is its standard

5 deviation, and $n_{p, h \in k}$ is the number of MR images belonging to the $k$ cohort. The

6 positive (negative) value of $Z_{p}^{\mathrm{CN}-\mathrm{AD}}$, for example, indicates that the distribution curve

7 of the average cortical thickness of the $\mathrm{CN}$ cohort is right (or left)-shifted compared to

8 that of $A D$ cohort. And the bigger the absolute value of the $Z$ score, the better

9 distinguished the distribution curves of average cortical thickness of the cohorts. In

10 this study, we identified ROI vertices satisfying the absolute value of the $Z$ score larger

11 than 1.5, and essential ROI vertices satisfying much higher cut-off Z scores (Table S1).

13 Construction of a statistical score matrix for classifying subjects into one 14 of $\mathrm{CN}, \mathrm{MCl}, \mathrm{AD}$ cohorts

15 Within each group of average cortical thickness, we constructed the statistical score 16 matrix for determining a subject's cohort as either $\mathrm{CN}, \mathrm{MCl}$, or $\mathrm{AD}$ [16]. First of all, $17 t_{p, h \in k}$ was transformed into the probability distribution matrix $P_{p, m}^{(k)}$, which is a 18 probability that the cortical thickness $t_{p, h \in k}$ at a vertex point $p$ of the subject in $k$ cohort 19 is between $(m-1) \Delta$ and $m \Delta$ :

$20 \quad P_{p, m}^{(k)}=\frac{\sum_{h \in k} \Theta(t-(m-1) \Delta) \cdot \Theta(m \Delta-t) \cdot \delta\left(t-t_{p, h}\right)}{\sum_{h \in k} 1}, m=1,2, \ldots 30, k=\mathrm{CN}, \mathrm{MCl}, \mathrm{AD}$.

21 Here, $\Delta=0.2 \mathrm{~mm}$, and cortical thickness index $m$ runs from 1 to 30 ; this covers the 
1 cortical thickness from $0 \mathrm{~mm}$ to $6 \mathrm{~mm}$. $\delta(x)$ is a Dirac delta function, and $\Theta(x)$ is a

2 step function where $\Theta(x<0)=0 ; \Theta(x \geq 0)=1$. Then, we defined the statistical score

3 matrix $S_{p, m}^{(k)}$ from $P_{p, m}^{(k)}$ in the following way:

$4 \quad S_{p, m}^{(k)}=-\ln \left[\frac{Q_{p, m}^{(k)}}{Q_{p}^{(k)}}\right], \quad Q_{p, m}^{(k)}=\frac{P_{p, m}^{(k)}}{\sum_{m} P_{p, m}^{(k)}}, \quad Q_{p}^{(k)}=\frac{\sum_{m} P_{p, m}^{(k)}}{\sum_{p} \sum_{m} P_{p, m}^{(k)}}$.

5 Since $\sum_{m} P_{p, m}^{(k)}=1, S_{p, m}^{(k)}=-\ln P_{p, m}^{(k)}-\ln \sum_{p} 1$ and the second term are constants. The

6 value of the statistical score matrix $S_{p, m}^{(k)}$ varies depending on the cohort $k$; the smaller

$7 S_{p, m}^{(k)}$ is, the larger $P_{p, m}^{(k)}$ is.

8 With this statistical score matrix $S_{p, m}^{(k)}$, we employed a strategy for determining to which

9 one of $k$ cohorts a given subject would belong. First, we evaluated the averaged

10 cortical thickness of a given subject over 276,825 vertices. Second, we assigned this

11 subject to one of four groups (A-D) of average cortical thickness. Third, based on the

12 preselected essential ROI vertices $p$ for the assigned group, we determined the

13 cortical thickness index m'(p) at which the cortical thickness at an essential ROI vertex

$14 p$ is between $(m-1) \Delta$ and $m \Delta$. Then, for each $k$ cohort, the total score $S_{(k)}^{\prime}$ was

15 calculated by summing up $S_{p, m_{\prime}^{\prime}(p)}^{(k)}$ over the preselected essential ROI vertices $p$ for

16 the assigned group of the average cortical thickness, $S_{(k)}^{\prime}=\sum_{p} S_{p, m \prime}^{(k)}(p)$. Lastly, to

17 which $k$ cohort a given subject would belong was decided by a cohort which gives the

18 minimum score out of $S^{\prime}(\mathrm{CN}), S^{\prime}(\mathrm{MCl}), \mathrm{S}^{\prime}(\mathrm{AD})$.

19 We, however, noted that the accuracy of both $P_{p, m}^{(A D)}$ and $S_{p, m}^{(A D)}$ may become 20 unsatisfactory if the number of people in the AD cohort was less than that of the $C N$ 21 cohort and the $\mathrm{MCl}$ cohort (Table 1). In order to overcome the unsatisfactory nature of 22 both $P_{p, m}^{(A D)}$ and $S_{p, m}^{(A D)}$, we employed the method of Kernel Density Estimation (KDE); 
1 namely, a Dirac delta function $\delta\left(t-t_{p, h}\right)$ in the definition of the probability distribution

2 matrix $P_{p, m}^{(k)}$, is replaced by a kernel function $f\left(\mathrm{t}-t_{p, h}\right)$ :

$3 f\left(t-t_{p, h}\right)=\sum_{l=-4}^{4} a_{l} \delta\left(t-\left(t_{p, h}+l \Delta\right)\right)$,

$4 \quad a_{l}=\operatorname{erf}(0.25+0.5|l|)+\frac{a_{0}}{2}-\sum_{l^{\prime}=0}^{|l|-1} a_{l^{\prime}}$.

5 Here, the relative ratio among coefficients $a_{l}$ is $a_{0}: a_{ \pm 1}: a_{ \pm 2}: a_{ \pm 3}: a_{ \pm 4}=56: 43: 21: 7: 1$.

6 The kernel function $f\left(\mathrm{t}-t_{p, h}\right)$ satisfies $\int_{-\infty}^{\infty} f\left(t-t_{p, h}\right) d t \approx 1$ and the standard 7 deviation $\sigma_{f} \approx 1.435$. Upon subjecting $\mathrm{KDE}, P_{p, m}^{(k)}$, becomes

$8 \quad P_{p, m}^{(k)}=\frac{\sum_{h \in k} \Theta(t-(m-1) \Delta) \cdot \Theta(m \Delta-t) \cdot f\left(t-t_{p, h}\right)}{\sum_{h \in k} 1}, m=1,2, \ldots 30, k=\mathrm{CN}, \mathrm{MCl}, \mathrm{AD}$.

9 In this study, we constructed the statistical score matrix on which KDE was employed

10 and used it for determining to which $k$ cohort a given subject would belong.

12 Construction of a covariance correlation matrix and a predictor for the

13 severity degree of AD

14 Within each group of the average cortical thickness, the severity degree of AD for a 15 given subject is estimated by the following strategy. First of all, we transformed the 16 cortical thickness matrix $t_{p, h}$ at essential ROI vertices $p$ for a subject $h$ into the 17 normalized matrix $t_{p, h}^{\prime}$ such that

$18 t_{p, h}^{\prime}=\frac{t_{p, h}-\left\langle t_{p, h}\right\rangle_{h}}{\sigma_{p}},\left\langle t_{p, h}\right\rangle_{h}=\frac{\sum_{h} t_{p, h}}{\sum_{h} 1}, \sigma_{p}^{2}=\left\langle t_{p, h}^{2}\right\rangle_{h}-\left\langle t_{p, h}\right\rangle_{h}^{2}$

19 Here, the product of $t_{p, j}^{\prime}$ by its transpose $t_{p, i}^{\prime}{ }^{T}$ results in the square matrix $t^{\prime \prime}{ }_{i j}=$ $20 t_{p, i}^{\prime}{ }^{T} \cdot t^{\prime}{ }_{p, j}$, and then its normalized matrix (called by a covariance correlation matrix) $21 C_{i j}$ is defined by $C_{i j}=t^{\prime \prime}{ }_{i j} / \max \left\{t^{\prime \prime}{ }_{i j}\right\}$, where $\max \left\{t^{\prime \prime}{ }_{i j}\right\}$ is the maximum value of 
1 elements in the square matrix $t^{\prime \prime}{ }_{i j}$. The larger the value of $C_{i j}$, the higher the

2 covariance correlation between a subject $i$ and a subject $j$ in their profile of the cortical

3 thickness at essential ROI vertices. Based on this covariance correlation matrix, we

4 defined the severity degree AD for a given subject $i$ by

$5 \quad S D_{i}=\left(\overline{C_{l}^{\mathrm{AD}-\mathrm{CN}}}-\left\langle\overline{C_{l}^{\mathrm{AD}-\mathrm{CN}}}\right\rangle_{i \in \mathrm{CN}}\right) /\left(\left\langle\overline{C_{l}^{\mathrm{AD}-\mathrm{CN}}}\right\rangle_{i \in \mathrm{AD}}-\left\langle\overline{C_{l}^{\mathrm{AD}-\mathrm{CN}}}\right\rangle_{i \in \mathrm{CN}}\right)$,

6 where $\overline{C_{l}^{\mathrm{AD}-\mathrm{CN}}}=\left\langle C_{i j}\right\rangle_{j \in \mathrm{AD}}-\left\langle C_{i j}\right\rangle_{j \in \mathrm{CN}}$. The severity degree of $\mathrm{AD}$ ranged from 0 for the

7 basin of $\mathrm{CN}$ state to 1 for the basin of $\mathrm{AD}$ state. Rank-ordering this degree in ascending 8 order illustrates that a subject $i$ with the larger (or smaller) value of the severity degree 9 is more prone to $A D(C N)$ state.

\section{Results}

12 Identification of essential ROI vertices at which the distributions of cortical thickness of $\mathrm{CN}, \mathrm{MCl}, \mathrm{AD}$ subjects are distinguishable

14 Although the averaged cortical thickness of subjects with $A D$ is generally known to be thinner than that of $\mathrm{CN}$ or $\mathrm{MCl}$ subjects, the distribution curves of averaged cortical thickness for the cohorts are not well distinguishable except near both ends of the

17 distribution curves as demonstrated in Fig 1A. This illustrates that a subject can be $\mathrm{CN}$ even though the averaged cortical thickness is thinner than that of a subject with AD, and vice versa. Also, we found that many subjects identified as $\mathrm{CN}, \mathrm{MCl}$, or $\mathrm{AD}$ have a similar averaged cortical thickness. This is due to the fact that the average cortical

21 thickness for a subject was calculated over all 276,825 vertices, and the cortical 22 thicknesses at most vertices are similar for all subjects, which prohibits us from successfully clustering 1552 human brain images into the image of $\mathrm{CN}, \mathrm{MCl}, \mathrm{AD}$ cohorts. Therefore, instead of resorting on the cortical thickness of all 276,825 vertices, 
1 we need to select the ROI vertices at which the cortical thickness values are

2 distinguishable from each other among $\mathrm{CN}, \mathrm{MCl}$, and $\mathrm{AD}$. For a fair selection of such

3 ROI vertices, we divided the range of the averaged cortical thickness of subjects into

4 four (A-D) different regions (for the detail, see the second section in methods).

5 Fig $1 \mathrm{~B}$ illustrates how we identified $\mathrm{ROI}$ and essential ROI vertices. The $\mathrm{x}$-axis

$6\left(\left\langle t_{p, h \in \mathrm{CN}}\right\rangle-\left\langle t_{p, h \in \mathrm{AD}}\right\rangle\right) / \sqrt{\sigma_{p, h \in \mathrm{CN}}^{2} / n_{p, h \in \mathrm{CN}}}$ represents the degree of separation between

7 the distribution curves of cortical thickness for $\mathrm{CN}$ subjects and $\mathrm{AD}$ subjects at a vertex

$8 p$ normalized by the dispersion of the cortical thickness of $\mathrm{CN}$ subjects, which is

9 quantified by the value of its $Z$ score. The $y$-axis

$10\left(\left\langle t_{p, h \in \mathrm{CN}}\right\rangle-\left\langle t_{p, h \in \mathrm{AD}}\right\rangle\right) / \sqrt{\sigma_{p, h \in \mathrm{AD}}^{2} / n_{p, h \in \mathrm{AD}}}$ represents values normalized by the

11 dispersion of the cortical thickness of individuals with AD. Therefore, the $x$-values $(y-$

12 values) at a vertex point $p$ represent the degree by which the distribution of cortical

13 thickness at this point $p$ of $\mathrm{CN}(\mathrm{AD})$ subjects is distinguished from averaged cortical

14 thickness of subjects with $A D(C N)$. It means the larger the value of $\left(Z_{p}^{\mathrm{CN}-\mathrm{AD}}\right)^{2}=$ $15\left[x^{-2}+y^{-2}\right]^{-1}$ is, the two distribution curves are more distinguished each other (for the 16 illustration, see Fig S1). The ROI cut-off line is defined by points satisfying $\left|Z_{p}^{\mathrm{CN}-\mathrm{AD}}\right|=$ 171.5 , and the distribution of cortical thickness of $\mathrm{CN}$ subjects and individuals with AD is 18 clearly distinguished at those points satisfying $\left|Z_{p}^{\mathrm{CN}-\mathrm{AD}}\right|>1.5$ (outside of the ROI cut19 off line).

20 Fig $1 \mathrm{C}$ shows ROI vertices for each of the cortical thickness groups (A-D) by colored 21 points on the white cortex, at which the thickness of the cortex is either thicker or 22 thinner particularly for one cohort compared with that of two other cohorts. These ROI 23 vertices are widely distributed on the cortex, and their locations are not fixed but vary 
1 depending on the groups $\mathrm{A}$ to $\mathrm{D}$. We uncovered, however, that the medial temporal

2 lobe, known to be very important for memory formation, is always indicated by a red

3 or dark red color irrespective of the groups A to D (Fig S2). This implies that the cortical

4 thickness values of the medial temporal lobe for subjects with AD are characteristically

5 thinner than those of $\mathrm{CN}$ subjects or individuals with $\mathrm{MCl}$ (red color), and this decrease

6 occurs in the following descending order: CN-MCI- AD (dark red color). The medial

7 temporal lobe is the region where the cortical thickness gradually decreases as

8 dementia progresses and therefore is the critical region necessary for determining the

9 dementia cohort and the severity degree of AD. We also noted that the cortical

10 thickness at the orange-colored region for subjects with $A D$ is thicker than that for $C N$

11 subjects or those with $\mathrm{MCl}$. This has nothing to do with the damage in the cortex but

12 contributes to the increase in the accuracy for predicting the dementia cohort since it

13 could provide better distinguishability of subjects with $A D$ from $C N$ subjects and those

14 with $\mathrm{MCl}$.

Character of statistical score matrix and classification of subject's cohort

17 The third section in methods described the detailed procedure of constructing the statistical score matrix for determining a subject's cohort within each group of the average cortical thickness (Fig 2A). In order to judge how well the statistical score matrix distinguishes $\mathrm{CN}, \mathrm{MCl}$, and $\mathrm{AD}$ cohorts from each other before we predict the

21 cohort of a new subject, we performed the singular value decomposition (SVD)

22 analysis on the combined statistical score matrix $S^{(\text {All) }}$ which consists of matrix 23 elements of $S^{(C N)}, S^{(M C l)}$, and $S^{(A D)}$. We used the SVD character of a matrix that a given 24 matrix can be reconstructed as a linear combination of the products of two singular 25 vectors by one singular matrix. Since the reconstructed matrix from the few highest 
1 modes of singular values contains the predominant character of an original given

2 matrix, one expects that the differences among the cohorts should appear in singular

3 vectors of different cohorts. Otherwise, the statistical score matrix $S^{(A l l)}$ is not reliable

4 nor does it contain the characteristic ingredient of different cohorts. Fig 2B, 2C and Fig

5 S3 show the highest six singular vectors corresponding to the six largest singular

6 values from SVD analysis of the statistical score matrices for each group of the

7 average cortical thickness $A$ to $D$. Here, it demonstrates that elements in the singular

8 vectors $v_{1}$ to $v_{3}$ for $\mathrm{CN}, \mathrm{MCl}$, and $\mathrm{AD}$ follow qualitatively a similar trend, meaning that

9 these compose the fundamental default modes, whereas those in $v_{4}$ to $v_{5}$ follow a 10 different trend and are distinguished each other.

11 Out of $547 \mathrm{CN}, 722 \mathrm{MCl}$, and $247 \mathrm{AD}$ human brain images predetermined clinically 12 and provided from the ADNI data set, we performed the self-recognition test and the 13 stratified 3-fold cross validation test using the 1516 human brain images for each 14 group on the average cortical thickness A to D (Table 2). For the first (second; third) 15 iteration of stratified 3-fold cross validation test, $1006(1011 ; 1015)$ human brain images were used as the training set for learning the statistical score matrix and 510 $17(505 ; 501)$ human brain images were used as an independent validation set. The new 18 method presented in this study recognized and predicted the subjects with $A D$ in the 19 cohort with more than $91 \%$ (self-recognition test) and $82 \%$ (stratified 3-fold cross 20 validation test) accuracy, respectively.

Table 2. Result for the tests of the cohorts for each group

\begin{tabular}{|c|c|c|c|c|c|c|c|c|c|c|}
\hline \multicolumn{11}{|c|}{ Self-recognition test } \\
\hline \multicolumn{2}{|r|}{ Group A } & \multicolumn{3}{|c|}{ Group B } & \multicolumn{3}{|c|}{ Group C } & \multicolumn{2}{|c|}{ Group D } & \multirow{2}{*}{ Correct (\%) } \\
\hline & $\mathrm{CN} \mathrm{MCl} \quad \mathrm{AD}$ & $\mathrm{CN}$ & $\mathrm{MCl}$ & $A D$ & $\mathrm{CN}$ & $\mathrm{MCl}$ & $A D$ & $\mathrm{CN}$ & $\mathrm{MCl} \quad \mathrm{AD}$ & \\
\hline
\end{tabular}




\begin{tabular}{c|cccccccccccc|cc}
$\mathrm{CN}$ & 128 & 8 & - & 197 & 12 & 3 & 142 & 11 & 6 & 40 & - & - & 507 & $(92)$ \\
$\mathrm{MCl}$ & 16 & 139 & - & 74 & 155 & 33 & 59 & 120 & 30 & 5 & 88 & 3 & 502 & $(69)$ \\
$\mathrm{AD}$ & - & - & 13 & 6 & 1 & 60 & 7 & 2 & 75 & 1 & 3 & 79 & 227 & $(91)$ \\
\hline
\end{tabular}

\section{Stratified 3-fold cross validation test}

\begin{tabular}{|c|c|c|c|c|c|c|c|c|c|c|c|c|c|}
\hline & \multicolumn{3}{|c|}{ Group A } & \multicolumn{3}{|c|}{ Group B } & \multicolumn{3}{|c|}{ Group C } & \multicolumn{3}{|c|}{ Group D } & \multirow{2}{*}{ Correct (\%) } \\
\hline & $\mathrm{CN}$ & $\mathrm{MCl}$ & $A D$ & $\mathrm{CN}$ & $\mathrm{MCl}$ & $A D$ & $\mathrm{CN}$ & $\mathrm{MCl}$ & $A D$ & $\mathrm{CN}$ & $\mathrm{MCl}$ & $A D$ & \\
\hline $\mathrm{CN}$ & 122 & 14 & - & 177 & 28 & 7 & 133 & 20 & 6 & 31 & 6 & 3 & $463 \quad(84)$ \\
\hline $\mathrm{MCl}$ & 33 & 122 & - & 97 & 130 & 35 & 68 & 103 & 38 & 9 & 75 & 12 & $430 \quad(59)$ \\
\hline AD & 1 & - & 12 & 5 & 9 & 53 & 7 & 11 & 66 & 2 & 8 & 73 & 204 (82) \\
\hline
\end{tabular}

"Exp." column, outside of the parenthesis, represents the number of MR images base on the clinical test, and "Score" row, inside of the parenthesis, represents the number of MR images base on our test using score matrix. AD, Alzheimer's disease; $C N$, cognitively normal; $\mathrm{MCl}$, mild cognitive impairment.

2 Estimating the severity of AD of subjects by covariance correlation matrix

3 Developing a quantitative measure to tell how serious the degree of dementia 4 progression for a given subject is very important for diagnosing and clinically treating 5 patients with $\mathrm{MCl}$ and $\mathrm{AD}$ with the different degree of Alzheimer dementia. In this study, 6 we already identified essential ROI vertices and constructed the statistical score

7 matrices as a classifier ensuring the correct prediction of subjects with AD at more 8 than $80 \%$ accuracy that they belong to the AD cohort. Thus, we extracted the cortical 9 thickness profile (or vector) at essential ROI vertices for all brain images, and 10 constructed the covariance correlation matrix between them. Then we compared the 11 profile for a given subject's image with that of patients with $A D$, to estimate the severity degree of $A D$ for a given subject towards patients with $A D$ (for the detail, see the fourth 
1 section in methods and Fig $3 \mathrm{~A}$ ). The personalized quantitative severity degree of

2 dementia (see the equation (8)) is plotted at the right-bottom graph of Fig. $3 \mathrm{~A}$ for each

3 subject of $C N, M C l, A D$ cohort of the group $D$ in the ascending order. The values of

4 severity degree of dementia were distributed around the averaged value of 0 (ranging

5 from about -0.5 to +0.5 ) for subjects with $\mathrm{CN}, 0.5$ (ranging from about -0.2 to +1.2 ) for

6 subjects with $\mathrm{MCl}$, and 1.0 (ranging from about +0.2 to +1.5 ) for subjects with $A D$,

7 respectively. The distribution of the severity degree for subjects with $\mathrm{MCl}$ was laid

8 across both ranges of those for $C N$ and $A D$, which points out that this is the intrinsic

9 source of the low success ratio in determining the dementia cohort of subjects with

$10 \mathrm{MCl}$. One could sort out quantitatively the broad spectrum of the severity of dementia

11 for $\mathrm{MCl}$ subjects in that whether they are prone to $\mathrm{CN}$ or how much they are

12 progressed toward AD. Given a new person for diagnosing the dementia state, one of

13 the cohort $\mathrm{CN}, \mathrm{MCl}, \mathrm{AD}$ was assigned by the equation (3) and the personalized

14 quantitative severity degree of dementia was estimated by the equation (8). Then, with

15 these two-qualitative and quantitative-determinants, one may infer that a new person

16 with the estimated severity degree below 0.0 is most likely to be $\mathrm{CN}$, with that between

170.0 and 0.5 might be $\mathrm{CN}$ or $\mathrm{MCl}$ prone to $\mathrm{CN}$, with that between 0.5 and 1.0 might be

$18 \mathrm{MCl}$ prone to $\mathrm{AD}$ or $\mathrm{AD}$, and with that above 1.0 is most likely to be $A D$ state.

19 We constructed the covariance correlation matrices for all groups A, B, C, D of the 20 average cortical thickness and observed the common pattern in the matrices that 21 subjects with $A D(C N)$, possessing a strong correlation among themselves are 22 clustered at the top-right (bottom-left) corner, represented by the cluster of red colors 23 (Fig S4). Also, we presented the reordered covariance correlation matrices by the 24 severity degree of $A D$ and the results to which one of the $C N, M C l, A D$ cohorts each 25 human brain would belong, based on both the clinical test and our stratified 3-fold 
1 cross validation test (Fig 3B). After comparing the result from our independent

2 validation test with that of the clinical test, we noted that those subjects which were

3 predicted to belong to the $\mathrm{MCl}$ cohort by the clinical test and yet estimated to have the

4 higher (lower) severity degree of AD by our estimation, were predicted to belong to the $5 \quad \mathrm{AD}(\mathrm{CN})$ cohort from the our validation test.

\section{Discussion}

8 Based on the cortical thickness data of 1516 human brain images from the ADNI data 9 set, we presented a new algebraic approach for both (1) the identification of the cohort $(\mathrm{CN}, \mathrm{MCl}, \mathrm{AD})$ a given subject would belong to and (2) the quantitative estimation of

11 the severity degree of $A D$ for a given new person (Fig 4). A total of 1516 human brain

12 MR images were partitioned into four groups by the average cortical thickness of each 13 subject. Out of 327,684 vertices on the cortex, a few hundred essential ROI vertices for each group were identified, which were enough to distinguish the cortical thickness distribution of the $\mathrm{CN}, \mathrm{MCl}$, and $\mathrm{AD}$ cohorts from each other. Statistical score matrices using the cortical thickness on the essential ROI vertices were constructed as a 17 classifier for determining the cohort of a given subject. Out of $547 \mathrm{CN}, 722 \mathrm{MCl}$, and $247 \mathrm{AD}$ subjects predetermined clinically, the success ratio for recognizing their cohort was $92 \%$ with $\mathrm{CN}, 69 \%$ with $\mathrm{MCl}$, and $91 \%$ with $\mathrm{AD}$ subjects. On the other hand, employing 1006 human brain images for control and 510 human brain images for

21 independent validation, the stratified 3-fold cross-validation test gave the correct 22 prediction rate of $84 \%$ with $\mathrm{CN}, 59 \%$ with $\mathrm{MCl}$, and $82 \%$ in subjects with $\mathrm{AD}$; this is in 23 the overall agreement with the results of clinical determination. Using the quantitative severity degree of $A D$ for subjects, we could explain the reason why the inevitable 
1 uncertainty in the determination of the $\mathrm{MCl}$ cohort arouse by the very broad distribution

2 of the severity degree of dementia which $\mathrm{MCl}$ subjects possess intrinsically. We

3 suggested that the severity degree of $A D$ presented in this study would be a realistic

4 measure for the quantitative personalized diagnosis of a given subject instead of tri-

5 partitioning the classification of a subject's cohort only by $\mathrm{CN}, \mathrm{MCl}$ or $\mathrm{AD}$. It is the

6 continuous degree for a given subject along the scale from 0 for the basin of $\mathrm{CN}$ state

7 to 1 for the basin of $A D$ state. One could sort out quantitatively the broad spectrum of

8 the severity degree of dementia for $\mathrm{MCl}$ or $\mathrm{AD}$ subjects with the different severity 9 degree of dementia in that whether they are prone to $\mathrm{CN}$ or how much they are 10 progressed toward AD.

\section{Limitations}

13 We noted above that the correct prediction rate of cohort for $\mathrm{MCl}$ subjects is $59 \%$ 14 which is lower than $84 \%, 82 \%$ for $C N$ and $A D$ subjects, respectively. This is because, as represented in Fig S2, there are no particular cortex regions in a brain, at which the cortical thickness is markedly different for $\mathrm{MCl}$ subjects compared to those for $\mathrm{CN}$ and AD subjects. Despite of such difficulties in predicting the cohort of subjects with $\mathrm{MCl}$, we introduced a new quantitative determinant "the severity degree of AD" so that we could identify $\mathrm{MCl}$ state by the quantitative manner as an intermediate one between

$20 \mathrm{CN}$ and $\mathrm{AD}$ states (Fig $3 \mathrm{~A}$ ). Therefore, instead of trying to single out $\mathrm{MCl}$ state as the one which is distinctively distinguished from $\mathrm{CN}$ and $\mathrm{AD}$ states, we focused on accessing how much a given subject with $\mathrm{MCl}$ possesses the similar character with

23 that of subjects with AD. Our ultimate mission in the future would be to verify how much

24 the severity degree of $A D$ for subjects with $\mathrm{MCl}$ will be correlated with the occurrence 
1 rate of $A D$ in a longitudinal study.

\section{Conclusions}

4 This study not only provided a straightforward algebraic approach to analyzing the

5 cortical thicknesses of human brains but also suggested quantitative measures by

6 which one could estimate both the cohort and the severity degree of AD for a given

7 new subject based on the neuro-images from the structural MRI. The MRI data of a 8 larger number of human brains could also be implemented into this study in a 9 systematic and robust manner, which would facilitate the better diagnose of AD with 10 the different degree of dementia.

\section{List of abbreviations}

14 AD, Alzheimer's Disease; ADNI, Alzheimer's Disease Neuroimaging Initiative; CN, 15 Cognitively Normal; E-bar, E-cohort color bar; KDE, Kernel Density Estimation; MCI, 16 Mild Cognitive Impairment; ROI, Region-Of-Interest; S-bar, S-cohort color bar; SVD, 17 Singular Value Decomposition

\section{Declarations}

\section{Acknowledgments}

22 We acknowledged DGIST supercomputing big data center for the allocation of 
1 supercomputing resources. We appreciated Mookyung Cheon and Wookyung Yu for

2 the fruitful discussions, and also Keonho Lee and Jangjae Lee of Chosun university

3 for the preparation of MRI image data in the initial stage of this work.

4

\section{Authors' contributions}

6 S. Kim and I. Chang designed the framework. S. Park executed the computation. S.

$7 \quad$ Kim and I. Chang analyzed the data and wrote the manuscript.

9 Funding

10 This work was supported by the National Research Foundation, Korea [grant number 11 NRF 2017R1E1A1A03070854].

13 Competing interests

14 All authors report no competing interests.

16 Availability of data and materials

17 Data used in the preparation of this article were obtained from the Alzheimer's Disease

18 Neuroimaging Initiative (ADNI) database (adni.loni.usc.edu). The ADNI was launched 19 in 2003 as a public-private partnership, led by principal investigator Michael W. Weiner, 20 MD. The primary goal of the ADNI has been to test whether serial MRI, PET, other 21 biological markers, and clinical and neuropsychological assessment can be combined 22 to measure the progression of $\mathrm{MCl}$ and early Alzheimer's disease. For up-to-date 23 information, see www.adni-info.org. 


\section{Consent for publication}

2 Not applicable.

3

\section{Ethics approval and consent to participate}

5 Data collection and sharing for this project was funded by the AD Neuroimaging

6 Initiative (ADNI) (National Institutes of Health Grant U01 AG024904) and DOD ADNI

7 (Department of Defense award number W81XWH-12-2-0012). ADNI is funded by the

8 National Institute on Aging, the National Institute of Biomedical Imaging and

9 Bioengineering, and through generous contributions from the following: AbbVie,

10 Alzheimer's Association; Alzheimer's Drug Discovery Foundation; Araclon Biotech;

11 BioClinica, Inc.; Biogen; Bristol-Myers Squibb Company; CereSpir, Inc.; Cogstate;

12 Eisai Inc.; Elan Pharmaceuticals, Inc.; Eli Lilly and Company; Eurolmmun; F.

13 Hoffmann-La Roche Ltd and its affiliated company Genentech, Inc.; Fujirebio; GE

14 Healthcare; IXICO Ltd.; Janssen Alzheimer Immunotherapy Research \& Development,

15 LLC.; Johnson \& Johnson Pharmaceutical Research \& Development LLC.; Lumosity;

16 Lundbeck; Merck \& Co., Inc.; Meso Scale Diagnostics, LLC.; NeuroRx Research;

17 Neurotrack Technologies; Novartis Pharmaceuticals Corporation; Pfizer Inc.; Piramal

18 Imaging; Servier; Takeda Pharmaceutical Company; and Transition Therapeutics. The

19 Canadian Institutes of Health Research is providing funds to support ADNI clinical

20 sites in Canada. Private sector contributions are facilitated by the Foundation for the

21 National Institutes of Health (www.fnih.org). The grantee organization is the Northern

22 California Institute for Research and Education, and the study is coordinated by the

23 Alzheimer's Therapeutic Research Institute at the University of Southern California.

24 ADNI data are disseminated by the Laboratory for Neuro Imaging at the University of

25 Southern California. 


\section{References}

4 1. Galvin JE, Sadowsky $\mathrm{CH}$, Nincds A. Practical guidelines for the recognition and 5 diagnosis of dementia. J Am Board Fam Med. 2012;25:367-82.

62 2. Solomon A, Soininen H. Dementia: Risk prediction models in dementia prevention. Nat 7

3. Raj A, Kuceyeski A, Weiner M. A network diffusion model of disease progression in

4. Hojjati SH, Ebrahimzadeh A, Babajani-Feremi A. Identification of the Early Stage of Alzheimer's Disease Using Structural MRI and Resting-State fMRI. Front Neurol.

5. Kim BH, Choi YH, Yang JJ, Kim S, Nho K, Lee JM, Alzheimer's Disease Neuroimaging 2019;10:904. Disease: Systems Biology Approach to Neuroimaging Endophenotype. J Alzheimers Dis. 2020.

6. Qiu S, Joshi PS, Miller MI, Xue C, Zhou X, Karjadi C, Chang GH, Joshi AS, Dwyer B, Zhu S, et al. Development and validation of an interpretable deep learning framework for Alzheimer's disease classification. Brain. 2020.

7. Tetreault AM, Phan T, Orlando D, Lyu I, Kang H, Landman B, Darby RR, Alzheimer's Disease Neuroimaging I. Network localization of clinical, cognitive, and neuropsychiatric symptoms in Alzheimer's disease. Brain. 2020;143:1249-60.

8. Hartikainen P, Rasanen J, Julkunen V, Niskanen E, Hallikainen M, Kivipelto M, Vanninen R, Remes AM, Soininen H. Cortical thickness in frontotemporal dementia, mild cognitive impairment, and Alzheimer's disease. J Alzheimers Dis. 2012;30:85774. 
$1 \quad$ 9. Im K, Lee JM, Seo SW, Yoon U, Kim ST, Kim YH, Kim SI, Na DL. Variations in cortical thickness with dementia severity in Alzheimer's disease. Neurosci Lett. 2008;436:22731.

10. Kim HJ, Ye BS, Yoon CW, Noh Y, Kim GH, Cho H, Jeon S, Lee JM, Kim JH, Seong JK, et al. Cortical thickness and hippocampal shape in pure vascular mild cognitive impairment and dementia of subcortical type. Eur J Neurol. 2014;21:744-51.

11. Lebedev AV, Westman E, Beyer MK, Kramberger MG, Aguilar C, Pirtosek Z, Aarsland D. Multivariate classification of patients with Alzheimer's and dementia with Lewy bodies using high-dimensional cortical thickness measurements: an MRI surfacebased morphometric study. J Neurol. 2013;260:1104-15.

12. Paternico D, Manes M, Premi E, Cosseddu M, Gazzina S, Alberici A, Archetti S, Bonomi E, Cotelli MS, Cotelli M, et al. Frontotemporal dementia and language networks: cortical thickness reduction is driven by dyslexia susceptibility genes. Sci Rep. 2016;6:30848.

13. Querbes O, Aubry F, Pariente J, Lotterie JA, Demonet JF, Duret V, Puel M, Berry I, Fort JC, Celsis P, Alzheimer's Disease Neuroimaging I. Early diagnosis of Alzheimer's disease using cortical thickness: impact of cognitive reserve. Brain. 2009;132:203647.

14. Dale AM, Fischl B, Sereno MI. Cortical surface-based analysis. I. Segmentation and surface reconstruction. Neuroimage. 1999;9:179-94.

15. Fischl B, Sereno MI, Dale AM. Cortical surface-based analysis. II: Inflation, flattening, and a surface-based coordinate system. Neuroimage. 1999;9:195-207.

16. Yu W, Lee W, Lee W, Kim S, Chang I. Uncovering symmetry-breaking vector and reliability order for assigning secondary structures of proteins from atomic NMR chemical shifts in amino acids. J Biomol NMR. 2011;51:411-24. 


\section{Figure legends}

4 Fig 1. The classification of 1516 brain images into four groups by the average cortical thickness. (A) The distribution of average cortical thickness of subjects in the

$\mathrm{CN}$ (black), $\mathrm{MCl}$ (cyan), and $\mathrm{AD}$ (magenta) cohorts. Above arrows point to the range

7 of average cortical thickness. Subject number, sex, and age for each group are listed

8 in Table 1. (B) For the cortical thickness group D, the degree of separation of the

9 distribution curve of average cortical thickness between $\mathrm{CN}$ subjects and AD subjects is presented in the form of black points. The closer to the origin point $(0,0)$ the degree

11 of separation of two distribution curves of average cortical thickness is, the less 12 distinguishable they are (Fig S1). Black points residing outside of the blue-dashed line $13(Z= \pm 1.5)$ are $\mathrm{ROI}$ vertices, and black points residing outside of red-dashed line $(Z$ values are listed in Table S1) are essential ROI vertices. (C) For each group of average cortical thickness, ROI vertices at which the thickness of the cortex for $\mathrm{CN}$ subjects is thicker (thinner) than that of the other subjects with $\mathrm{MCl}$ or $\mathrm{AD}$ is represented by cyan

17 (blue) color. As a similar procedure, $\mathrm{ROI}$ vertices for $\mathrm{MCl}$ subjects is thicker (thinner) 18 than the other cohorts is represented by green (dark green) color. Also, ROI vertices 19 for $A D$ subjects is thicker (thinner) than the other cohorts is represented by orange 20 (red) color. Especially, the ROI vertices at which the cortical thickness decreases in 21 the descending order of $\mathrm{CN}-\mathrm{MCl}-\mathrm{AD}$ is represented by dark red. And the essential ROI 22 vertices are represented by a black color. The ROI vertices commonly found from more 23 than three groups of average cortical thickness are presented in Fig S2. 
1 Fig 2. The character of score matrix for each group of average cortical thickness.

2 (A) For a given group of average cortical thickness, three kinds of heat maps illustrate

3 the process starting from the cortical thickness matrix at all 276,825 vertices to that at

4 only 564 essential ROI vertices, and then construction of the score matrix. The

5 dimension in the x-axis of the cortical thickness matrix at all 276,825 is too large to

6 draw, we placed the blank in the middle to abbreviate the large dimension of the $x-$

7 axis. (B) The results of singular value decomposition analysis on score matrices, which

8 are composed of $547 \mathrm{CN}, 722 \mathrm{MCl}, 247 \mathrm{AD}$ human brain images and used for self-

9 recognition test. For each group of average cortical thickness, six singular vectors

10 corresponding to the six largest singular values are presented. Here, $x$-axis is $m$ value

11 defined in the third section of methods, and y-axis is an arbitrary unit for the singular

12 vectors. For each graph, the singular vector components for $\mathrm{CN}, \mathrm{MCl}$, and $\mathrm{AD}$ subjects are plotted by black, cyan, magenta colors, respectively. (C) The results of singular value decomposition analysis on score matrices, which are composed of $363 \mathrm{CN}, 480$ $\mathrm{MCl}, 163 \mathrm{AD}$ human brain images as a training set and used for the first iteration of the stratified 3-fold cross validation test. The other results of that used for the second and third iterations of the stratified 3-fold cross validation test are presented in Fig S3.

Fig 3. Covariance correlation matrix and severity degree of AD. (A) The left-top heat map is the covariance correlation matrix for group D of average cortical thickness.

21 The $\mathrm{x}$ - and $\mathrm{y}$-axes represent the indices 1 to 40 for $\mathrm{CN}$ subjects, 41 to 136 for $\mathrm{MCl}$ 22 subjects, and 137 to 219 for AD subjects. Here, red (blue) color represents the high 23 (low) correlation between two subjects at the essential ROI vertices. The extra Ecohort color bar at the right of the heat map represents the clinically determined cohort of $\mathrm{CN}$ subjects and subjects with $\mathrm{MCl}$ and $\mathrm{AD}$ denoted by black, cyan, and magenta 
1 colors, respectively. The left-bottom graph illustrates the personalized severity degree

2 of dementia for each subjects of the group $D$ in terms of a quantitative value, ranging

3 from 0 for the basin of $\mathrm{CN}$ state to 1 for the basin of $A D$ state (for the detail, see the

4 fourth section in methods). The average values of this severity degree in each cohort

5 are denoted by horizontal lines, respectively. The left panel is reordered into the right

6 panel according to the ascending value of the severity degree in each cohort. For

7 those subjects with $\mathrm{MCl}$, the distribution of severity degree of dementia is very broad.

8 One can sort out the broad spectrum of the severity of dementia for $\mathrm{MCl}$ subjects in

9 that whether they are prone to $\mathrm{CN}$ or how much they are progressed toward AD. (B)

10 The reordered covariance correlation matrices for A, B, C, and D groups of average

11 cortical thickness together with the determination of $\mathrm{CN}$ (black), $\mathrm{MCl}$ (cyan), and $\mathrm{AD}$

12 (magenta) cohorts by clinical (E-cohort color bar) exam and by the stratified 3-fold 13 cross validation test of this study (S-cohort color bar). The original covariance 14 correlation matrices for the four groups of average cortical thickness are provided in 15 Fig S4.

17 Fig 4. Flow chart for the cohort determination and the estimation of the severity 18 degree of $\mathbf{A D}$. The left shows the process of constructing the score matrix and 19 covariance correlation matrix from the cortical thickness big data of subjects derived 20 from the ADNI data set. The right shows the process of determining the cohort and the 21 severity degree of $A D$ for a new given subject. 
Figures
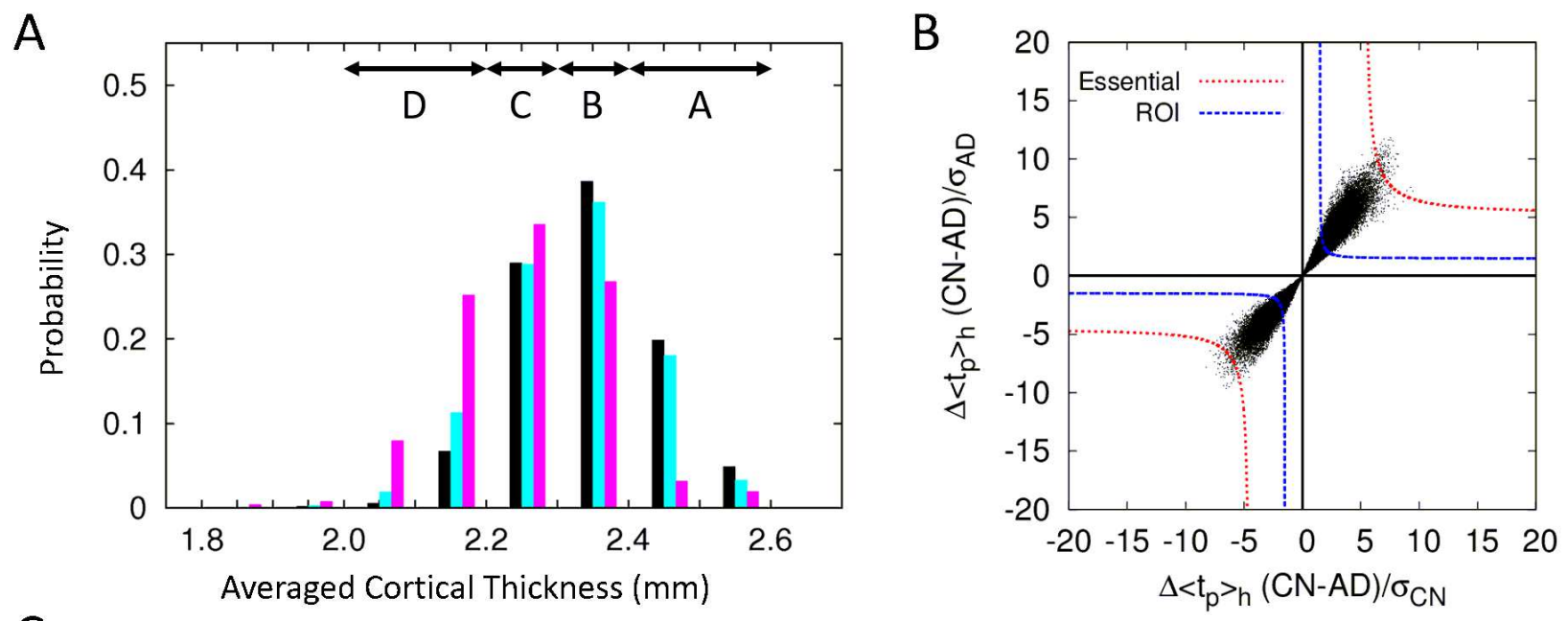

C
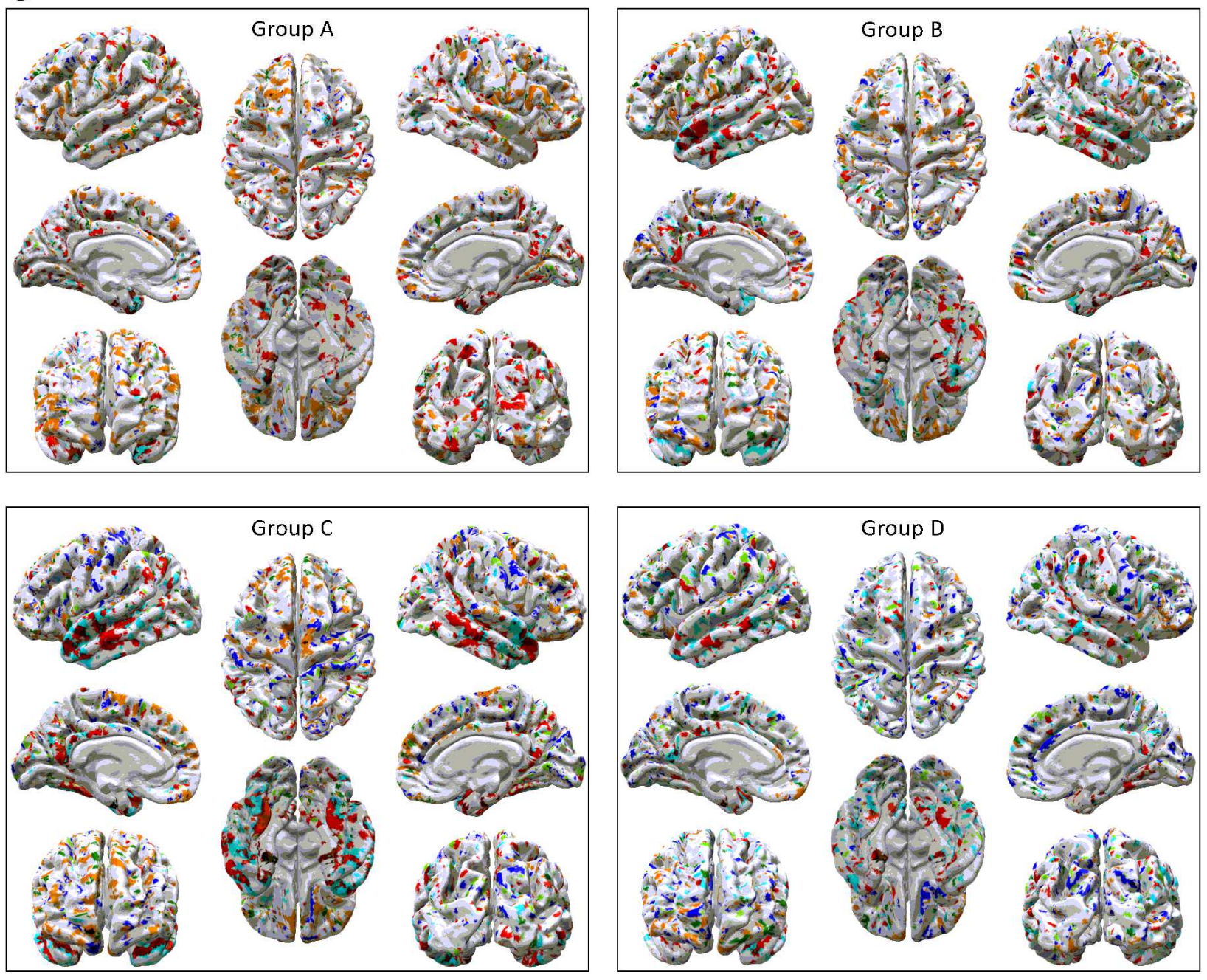

Figure 1

The classification of 1516 brain images into four groups by the average cortical thickness. (A) The distribution of average cortical thickness of subjects in the $\mathrm{CN}$ (black), $\mathrm{MCl}$ (cyan), and $\mathrm{AD}$ (magenta) cohorts. Above arrows point to the range of average cortical thickness. Subject number, sex, and age for 
each group are listed in Table 1. (B) For the cortical thickness group D, the degree of separation of the distribution curve of average cortical thickness between $\mathrm{CN}$ subjects and $\mathrm{AD}$ subjects is presented in the form of black points. The closer to the origin point $(0,0)$ the degree of separation of two distribution curves of average cortical thickness is, the less distinguishable they are (Fig S1). Black points residing outside of the blue-dashed line $(Z= \pm 1.5)$ are ROI vertices, and black points residing outside of reddashed line (Z values are listed in Table S1) are essential ROI vertices. (C) For each group of average cortical thickness, ROI vertices at which the thickness of the cortex for $\mathrm{CN}$ subjects is thicker (thinner) than that of the other subjects with $\mathrm{MCl}$ or $\mathrm{AD}$ is represented by cyan (blue) color. As a similar procedure, $\mathrm{ROI}$ vertices for $\mathrm{MCl}$ subjects is thicker (thinner) than the other cohorts is represented by green (dark green) color. Also, ROI vertices for AD subjects is thicker (thinner) than the other cohorts is represented by orange (red) color. Especially, the ROI vertices at which the cortical thickness decreases in the descending order of CN-MCl-AD is represented by dark red. And the essential ROI vertices are represented by a black color. The ROI vertices commonly found from more than three groups of average cortical thickness are presented in Fig S2. 
A

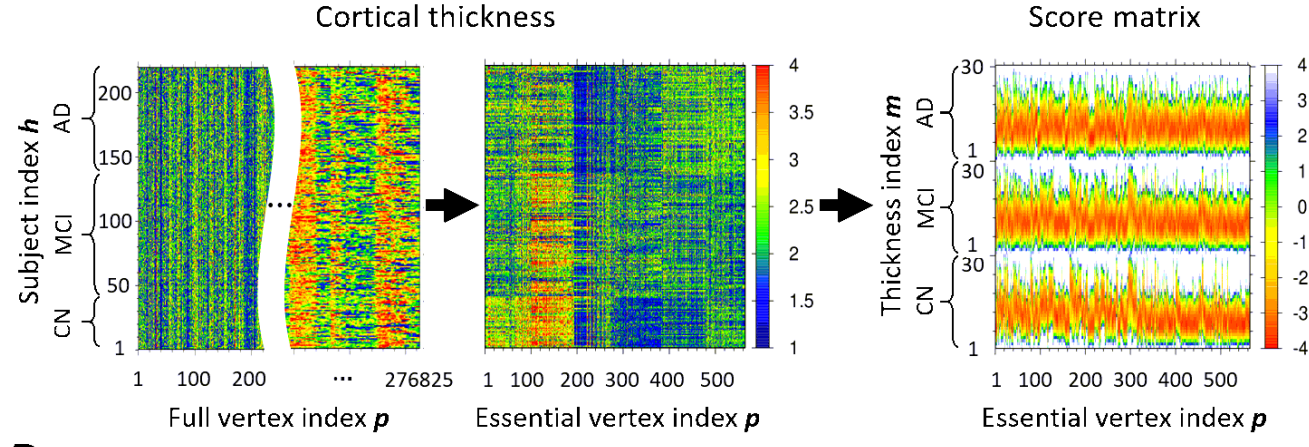

B

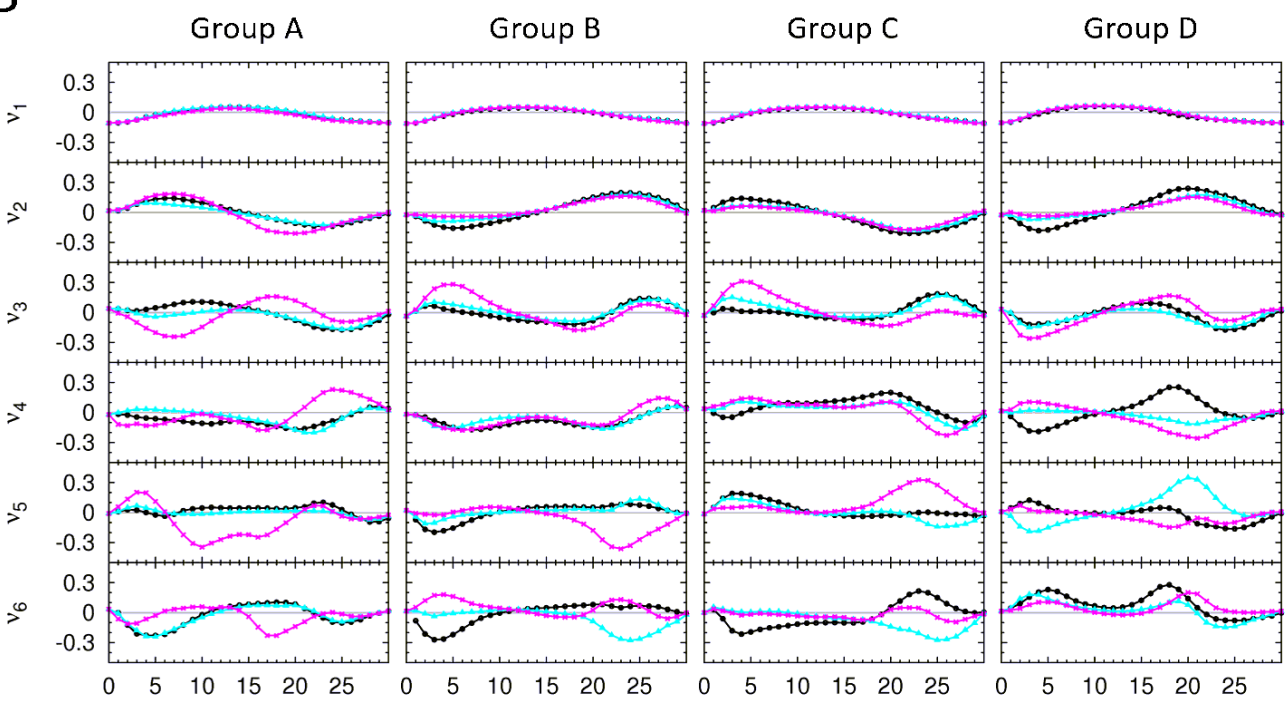

$\mathrm{C}$

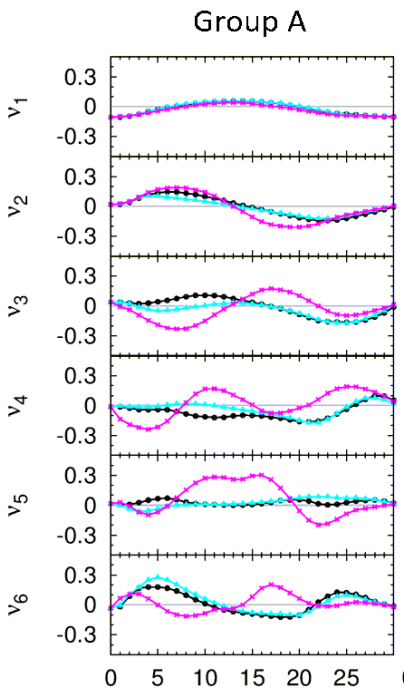

Group B

Group C

Group D

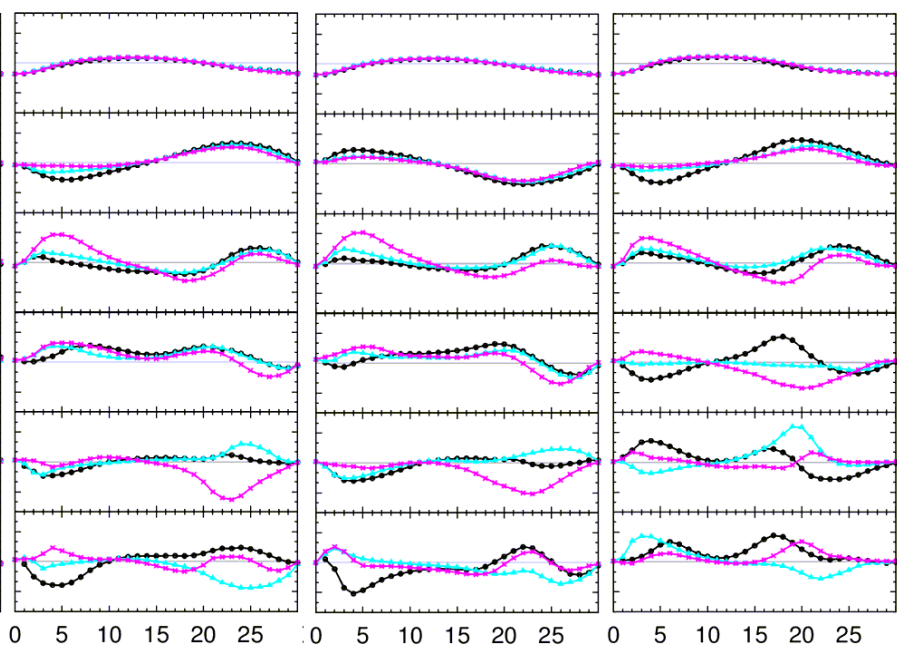

Figure 2

The character of score matrix for each group of average cortical thickness. (A) For a given group of average cortical thickness, three kinds of heat maps illustrate the process starting from the cortical thickness matrix at all 276,825 vertices to that at only 564 essential ROI vertices, and then construction of the score matrix. The dimension in the $x$-axis of the cortical thickness matrix at all 276,825 is too large to draw, we placed the blank in the middle to abbreviate the large dimension of the x-axis. (B) The results of 
singular value decomposition analysis on score matrices, which are composed of $547 \mathrm{CN}, 722 \mathrm{MCl}, 247$ $A D$ human brain images and used for self-recognition test. For each group of average cortical thickness, six singular vectors corresponding to the six largest singular values are presented. Here, $x$-axis is $m$ value defined in the third section of methods, and $y$-axis is an arbitrary unit for the singular vectors. For each graph, the singular vector components for $\mathrm{CN}, \mathrm{MCl}$, and $\mathrm{AD}$ subjects are plotted by black, cyan, magenta colors, respectively. (C) The results of singular value decomposition analysis on score matrices, which are composed of $363 \mathrm{CN}, 480 \mathrm{MCl}, 163 \mathrm{AD}$ human brain images as a training set and used for the first iteration of the stratified 3-fold cross validation test. The other results of that used for the second and third iterations of the stratified 3-fold cross validation test are presented in Fig S3. 

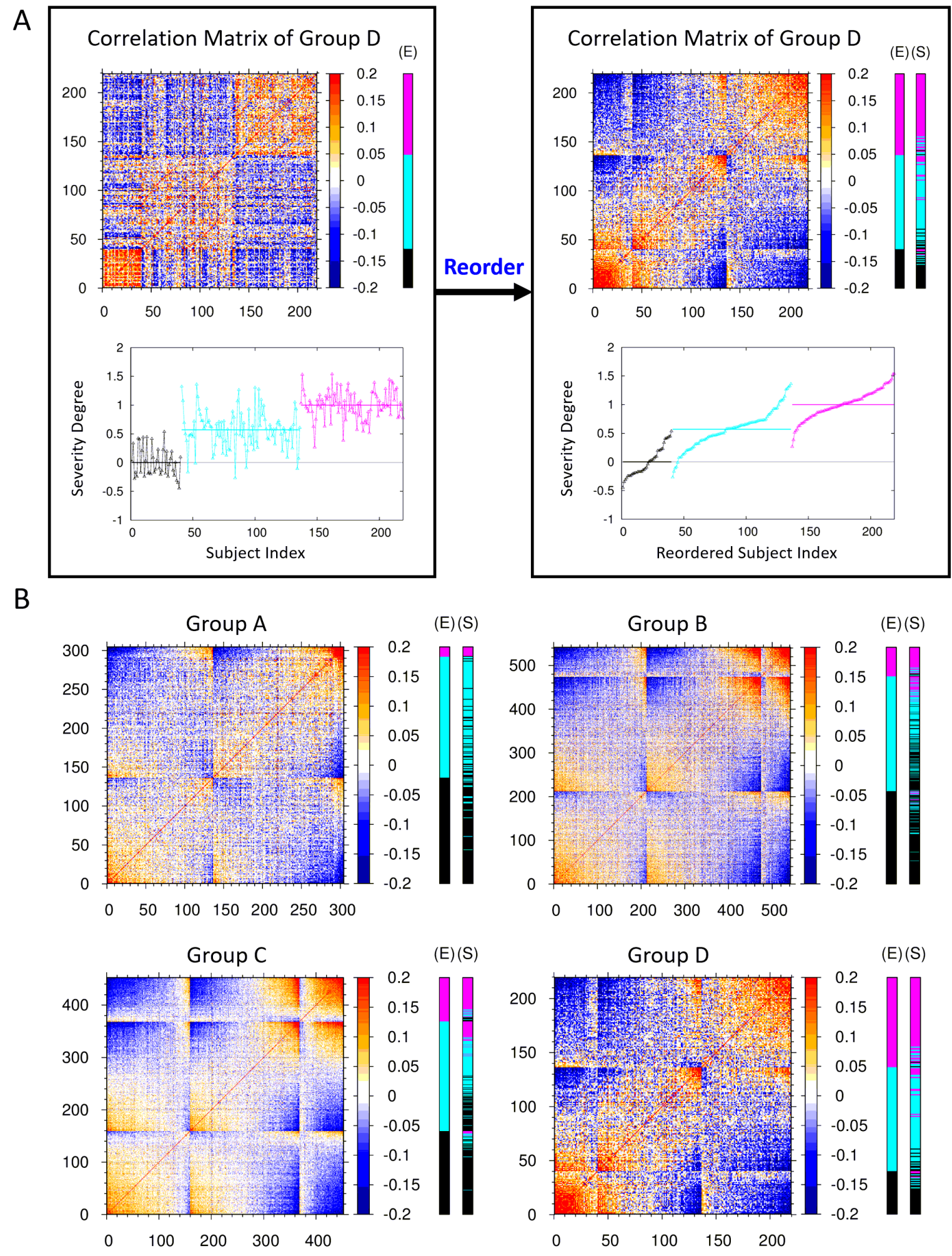

\section{Figure 3}

Covariance correlation matrix and severity degree of AD. (A) The left-top heat map is the covariance correlation matrix for group $D$ of average cortical thickness. The $x$-and $y$-axes represent the indices 1 to 40 for $\mathrm{CN}$ subjects, 41 to 136 for $\mathrm{MCl}$ subjects, and 137 to 219 for AD subjects. Here, red (blue) color represents the high (low) correlation between two subjects at the essential ROI vertices. The extra Ecohort color bar at the right of the heat map represents the clinically determined cohort of $\mathrm{CN}$ subjects 
and subjects with $\mathrm{MCl}$ and $\mathrm{AD}$ denoted by black, cyan, and magenta colors, respectively. The left-bottom graph illustrates the personalized severity degree of dementia for each subjects of the group $D$ in terms of a quantitative value, ranging from 0 for the basin of $C N$ state to 1 for the basin of $A D$ state (for the detail, see the fourth section in methods). The average values of this severity degree in each cohort are denoted by horizontal lines, respectively. The left panel is reordered into the right panel according to the ascending value of the severity degree in each cohort. For those subjects with $\mathrm{MCl}$, the distribution of severity degree of dementia is very broad. One can sort out the broad spectrum of the severity of dementia for $\mathrm{MCl}$ subjects in that whether they are prone to $\mathrm{CN}$ or how much they are progressed toward $A D$. (B) The reordered covariance correlation matrices for $A, B, C$, and D groups of average cortical thickness together with the determination of $\mathrm{CN}$ (black), $\mathrm{MCl}$ (cyan), and $\mathrm{AD}$ (magenta) cohorts by clinical (E-cohort color bar) exam and by the stratified 3-fold cross validation test of this study (S-cohort color bar). The original covariance correlation matrices for the four groups of average cortical thickness are provided in Fig S4. 

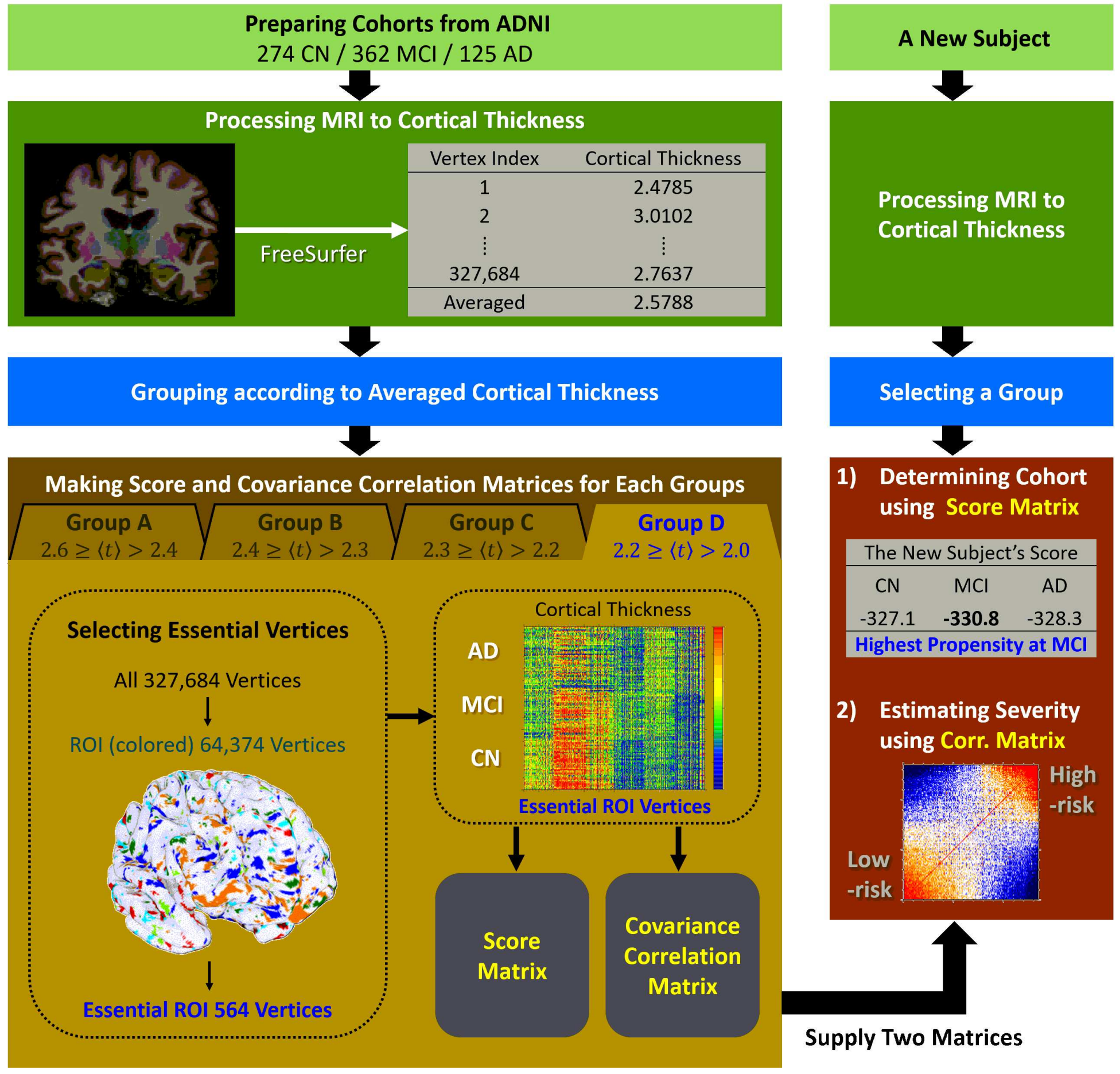

\section{1) Determining Cohort using Score Matrix}

The New Subject's Score

$\begin{array}{llll}\mathrm{CN} & \mathrm{MCl} & \mathrm{AD}\end{array}$

$\begin{array}{lll}-327.1 & -330.8 & -328.3\end{array}$

Highest Propensity at $\mathrm{MCl}$

2) Estimating Severity using Corr. Matrix
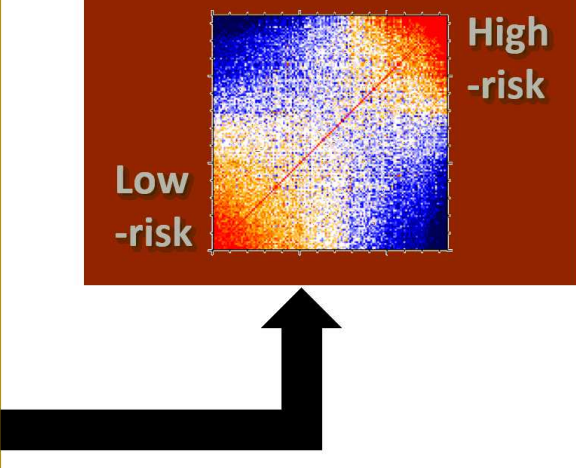

Supply Two Matrices

Figure 4

Flow chart for the cohort determination and the estimation of the severity degree of AD. The left shows the process of constructing the score matrix and covariance correlation matrix from the cortical thickness big data of subjects derived from the ADNI data set. The right shows the process of determining the cohort and the severity degree of AD for a new given subject.

\section{Supplementary Files}


This is a list of supplementary files associated with this preprint. Click to download.

- Supplementaryinformation20201201.docx 